\title{
Pinning and collective modes of a vortex lattice in a Bose-Einstein condensate
}

\author{
J.W. Reijnders ${ }^{1, *}$ and R.A. Duine ${ }^{2, \dagger}$ \\ ${ }^{1}$ Institute for Theoretical Physics, University of Amsterdam, \\ Valkenierstraat 65, 1018 XE Amsterdam, The Netherlands \\ ${ }^{2}$ The University of Texas at Austin, Department of Physics, \\ 1 University Station C1600, Austin, TX 78712-0264
}

(Dated: October 28, 2018)

\begin{abstract}
We consider the ground state of vortices in a rotating Bose-Einstein condensate that is loaded in a co-rotating two-dimensional optical lattice. Due to the competition between vortex interactions and their potential energy, the vortices arrange themselves in various patterns, depending on the strength of the optical potential and the vortex density. We outline a method to determine the phase diagram for arbitrary vortex filling factor. Using this method, we discuss several filling factors explicitly. For increasing strength of the optical lattice, the system exhibits a transition from the unpinned hexagonal lattice to a lattice structure where all the vortices are pinned by the optical lattice. The geometry of this fully-pinned vortex lattice depends on the filling factor and is either square or triangular. For some filling factors there is an intermediate half-pinned phase where only half of the vortices is pinned. We also consider the case of a two-component Bose-Einstein condensate, where the possible coexistence of the above-mentioned phases further enriches the phase diagram. In addition, we calculate the dispersion of the low-lying collective modes of the vortex lattice and find that, depending on the structure of the ground state, they can be gapped or gapless. Moreover, in the half-pinned and fully-pinned phase, the collective mode dispersion is anisotropic. Possible experiments to probe the collective mode spectrum, and in particular the gap, are suggested.

PACS numbers: 03.75.Kk, 67.40.-w, 32.80.Pj
\end{abstract}

${ }^{*}$ Electronic address: jwr@science.uva.nl

$\dagger$ Electronic address: duine@physics.utexas.edu URL: http://www.ph.utexas.edu/〜duine 


\section{INTRODUCTION}

It has been known since the work of Onsager [1] and Feynman [2] that a superfluid supports angular momentum only through quantized vortices. Furthermore, following Abrikosov's prediction that vortices in type-II superconductors arrange themselves on a lattice [3], and its experimental confirmation [4, 5], Tkachenko showed that vortex lines in a rotating superfluid form a regular hexagonal lattice in the absence of disorder [6]. Such an Abrikosov lattice, as it is nowadays called, was indeed observed experimentally [7, 8]. Tkachenko also predicted the vortex lattices to support phonons, the so-called Tkachenko modes [9].

With the first experimental realization of Bose-Einstein condensation in ultracold dilute atomic gases [10], another regime in the physics of neutral superfluids has become accessible, i.e., the weakly-interacting regime. Following this achievement, the same group created, for the first time, a vortex in an atomic Bose-Einstein condensate [11]. Although there has been some experimental interest in the equilibrium and nonequilibrium behavior of a single

vortex line [12, 13, 14], since the observation of a Bose-Einstein condensate with more than one vortex [15], however, most of the experimental studies are focused on vortex lattices [16, 17, 18]. In particular, the dependence of the lowest Tkanchenko mode on the rotation frequency has been measured [19], and is theoretically well understood [20].

One aspect that distinguishes the physics of vortices in atomic Bose-Einstein condensates from superfluid Helium and superconductors, is that in the latter systems the pinning of vortices due to intrinsic disorder in the system plays an important role [21, 22, 23, 24, 25, 26, 27]. This, together with the discovery of high-temperature superconductors, has led to many theoretical studies of the effects of pinning on the melting of the vortex lattice 28, 29, 30, 31]. Furthermore, in the context of type-II superconductivity, there has been a lot of interest in the effects of a periodic array of pinning centers on the ground state of the vortices [32, 33, 34, 35, 36]. In particular, it turns out that, due to the competition between vortex interactions and pinning, the system exhibits a rich ground state phase diagram, as a function of the vortex density and the strength of the pinning potential [36]. However, since the pinning potential in the case of vortices in type-II superconductors is known only phenomenologically, a detailed comparison between theory and experiment seems unfeasible.

Very recently, we have shown that a rotating Bose-Einstein condensate in a so-called 
optical lattice is a very attractive system to study the pinning of vortex lattices in a superfluid 37]. Such an optical lattice is formed by laser fields that trap the atoms using the dipole force. Recently, the experimental control over the strength of the optical lattice enabled Greiner et al. [38] to experimentally explore the Mott-Insulator to superfluid quantum phase transition [39, 40]. By rotating the optical lattice at the same frequency of rotation as the Bose-Einstein condensate, the vortices experience a static pinning potential that is determined by the optical lattice [37, 41, 42]. Such a co-rotating optical lattice can be made by rotating holographic phase plates or amplitude masks [43, 44, 45, 46]. Since the strength of the optical lattice determines the strength of the pinning potential, and the rotation frequency controls the density of vortices, the phase diagram can be studied in detail experimentally.

In Ref. [37] we have calculated the phase diagram for a homogenous Bose-Einstein condensate with one vortex per unit cell of the optical lattice analytically, by means of a variational method. It is the aim of this paper to extend these calculations to other vortex filling factors and to the situation of a two-component Bose-Einstein condensate. Furthermore, we also study the collective modes of the pinned and unpinned vortex lattices. Complementary to our analytical work, $\mathrm{Pu}$ et al. [47] numerically studied a Bose-Einstein condensate in a co-rotating optical lattice with an additional harmonic confining potential. The harmonic trapping potential leads to finite-size effects which further enrich the phase diagram of the system. Unfortunately, including an additional harmonic potential in our variational calculations makes analytical results unfeasible. Therefore, we consider the homogeneous case, which brings out the physics of the competition between vortex interactions and pinning potential most clearly. In Ref. [37] we studied both the case of a one-dimensional optical lattice and the two-dimensional case. In this paper we focus on the two-dimensional situation.

The paper is organized as follows. In Sec. II we derive the pinning potential and vortex interaction energy. Using these results, we calculate in Sec. III the energy of an arbitrary vortex lattice in a periodic potential. This result is used to determine the ground state phase diagram in Sec. IV for a single-component Bose-Einstein condensate for various filling factors. The two-component case is discussed in Sec. V] In Sec. VI we determine the dispersion of the low-lying collective modes over the ground state. We end in Sec. VII with our conclusions. 


\section{VORTEX INTERACTIONS AND POTENTIAL ENERGY}

In this section we calculate the interaction energy of two vortices, as well as the potential energy of a vortex in the optical lattice, i.e., the pinning potential, by means of a variational ansatz. These results are needed later on to determine the phase diagram.

\section{A. Pinning potential}

Since we assume the system to be at zero temperature throughout the paper, the most convenient starting point is the hamiltonian functional which gives the total energy of the system in terms of the macroscopic condensate wave function $\Psi(\mathbf{x})$, and reads

$$
H\left[\Psi^{*}, \Psi\right]=\int d \mathbf{x} \Psi^{*}(\mathbf{x})\left[-\frac{\hbar^{2} \nabla^{2}}{2 M}+\frac{1}{2} g|\Psi(\mathbf{x})|^{2}+V_{\mathrm{OL}}(\mathbf{x})-\mu\right] \Psi(\mathbf{x}) .
$$

Here, $M$ denotes the mass of one atom which interacts with the other atoms via a two-body contact interaction of strength $g=4 \pi a_{s} \hbar^{2} / M$, with $a_{s}>0$ the $s$-wave scattering length. The two-dimensional optical lattice potential is given by

$$
V_{\mathrm{OL}}(\mathbf{x})=s E_{\mathrm{R}}\left[\sin ^{2}(q x)+\sin ^{2}(q y)\right]
$$

with $E_{\mathrm{R}}$ the recoil energy, $q$ the wavenumber of the optical lattice, and $s \geq 0$ a dimensionless number indicating the strength of the optical lattice. The chemical potential that fixes the number of atoms in the condensate is given by $\mu$.

Throughout this paper we consider for simplicity a condensate with infinite extent in the $x$ - $y$-plane which is tightly confined in the $z$-direction by an harmonic trap with frequency $\omega_{z}$. This approach is motivated by the fact that a Bose-Einstein condensate that is rotated around the $z$-axis will extend in the $x$-y-plane due to the centrifugal force. Assuming that modes in the $z$-direction are frozen out, such that the wave function is gaussian in this direction, effectively leads to a condensate thickness $d_{z} \equiv \sqrt{\pi \hbar /\left(M \omega_{z}\right)}$. These assumptions allow us to neglect the curvature of the vortex lines along the $z$-direction. Note also that we can safely omit the term proportional to the external rotation frequency in Eq. (II), since we intend to work with a variational ansatz which has a fixed vortex density, and, moreover, we assume that the harmonic magnetic trapping potential approximately cancels the centrifugal force. 
We consider the system in the Thomas-Fermi limit where the kinetic energy of the condensate atoms is neglected with respect to their potential energy and mean-field interaction energy. Minimizing the hamiltonian of Eq. (11) in this limit, the global density profile of the condensate without vortices is given by

$$
n_{\mathrm{TF}}(\mathbf{x})=|\Psi(\mathbf{x})|^{2}=n-\left[V_{\mathrm{OL}}(\mathbf{x})-s E_{\mathrm{R}}\right] / g
$$

with $n=\left[\mu-s E_{\mathrm{R}}\right] / g$ the average density of the condensate.

As already mentioned, to find the potential energy of a vortex in a Bose-Einstein condensate in an optical lattice, as a function of its coordinates $\left(u_{x}, u_{y}\right)$, we use a variational ansatz for the condensate wave function. It is given by

$$
\Psi(\mathbf{x})=\sqrt{n_{\mathrm{TF}}(\mathbf{x})} \Theta[|\mathbf{x}-\mathbf{u}| / \xi-1] \exp [i \phi(\mathbf{x}, \mathbf{u})]
$$

with $\xi=1 / \sqrt{8 \pi a_{s} n}$ the healing length that sets the size of the vortex core, $\phi(\mathbf{x}, \mathbf{u})=$ $\arctan \left[\left(y-u_{y}\right) /\left(x-u_{x}\right)\right]$ the phase configuration corresponding to one vortex, and $\Theta(z)$ the unit step function. For the above ansatz to be a good approximation, we have assumed that the vortex core is much smaller then an optical lattice period, $q \xi \ll 1$, and that the strength of the potential is sufficiently weak, $s E_{\mathrm{R}}<\mu$. The use of a unit step function for the density profile of the vortices is justified because the main contribution to the energy of the vortices is due to the superfluid velocity pattern and not due to the inhomogeneity of the condensate density [48].

Substituting the ansatz in Eq. (41) in the hamiltonian in Eq. (11) and integrating over the entire $x$-y-plane gives the total energy of the vortex in the optical lattice. This energy diverges with the system size. However, we need to isolate the finite, position dependent contribution to the energy due to the presence of the vortex, which is the only relevant contribution for our purposes.

There are two position dependent terms which contribute significantly to the energy. The first one is largest and is entirely due to the kinetic energy of the condensate. Neglecting the effect of the laplacian on the global density profile, which is consistent with the ThomasFermi limit, we have

$$
U_{\text {kin }}\left(u_{x}, u_{y}\right)=-\frac{d_{z} s E_{\mathrm{R}}}{8 a_{s}} \int d x d y\left[\frac{\sin ^{2}(q x)+\sin ^{2}(q y)}{\left(x-u_{x}\right)^{2}+\left(y-u_{y}\right)^{2}}\right] .
$$


The integral can be done by shifting the integration variables to $x=\rho \cos \theta+u_{x}$ and $y=\rho \sin \theta+u_{y}$. A little algebra yields

$$
\begin{aligned}
U_{\text {kin }}\left(u_{x}, u_{y}\right)= & \frac{d_{z} s E_{\mathrm{R}}}{8 a_{s}}\left[\cos \left(2 q u_{x}\right)+\cos \left(2 q u_{y}\right)\right] \int_{0}^{2 \pi} d \theta \int_{\xi}^{\infty} \frac{d \rho}{2 \rho} \cos (2 q \rho \cos \theta) \\
& -\frac{d_{z} s E_{\mathrm{R}}}{8 a_{s}}\left[\sin \left(2 q u_{x}\right)+\sin \left(2 q u_{y}\right)\right] \int_{0}^{2 \pi} d \theta \int_{\xi}^{\infty} \frac{d \rho}{2 \rho} \sin (2 q \rho \sin \theta) .
\end{aligned}
$$

When integrated over polar angle, the second part on the righthand side of this expression gives zero. The integral in the remaining part can be further simplyfied by using a Jacobi expansion and integrating out the polar angle

$$
\sum_{n=-\infty}^{\infty}(-1)^{n} \int_{\xi}^{\infty} \frac{d \rho}{2 \rho} J_{2 n}(2 q \rho) \int_{0}^{2 \pi} d \theta e^{2 i n \theta}=\pi \int_{\xi}^{\infty} \frac{d \rho}{\rho} J_{0}(2 \rho q) \equiv \pi Q_{\mathrm{kin}}(q \xi)
$$

where $J_{l}$ is the $l$-th orther Bessel function of the first kind.

The second vortex position dependent contribution to the energy comes solely from the core. Let us consider the energy contribution

$$
U=d_{z} \int d^{2} x \Psi^{*}(\mathbf{x})\left[V_{\mathrm{OL}}(\mathbf{x})+\frac{g}{2}|\Psi(\mathbf{x})|^{2}-\mu\right] \Psi(\mathbf{x})
$$

Alternatively, this term is written as $U=E_{\infty}-U_{\text {core }}(\mathbf{u})$, where $E_{\infty}$ is a divergent constant equal to the energy of the condensate without a vortex and $U_{\text {core }}(\mathbf{u})$ the contribution of the region excluded by the core of the vortex. Since the latter depends on the vortex coordinates this contribution must be taken into account which gives

$$
\begin{aligned}
U_{\text {core }}(\mathbf{u}) & =-d_{z} \int_{\text {core }} d^{2} x\left[\frac{g}{2} n_{\mathrm{TF}}(\mathbf{x}) n_{\mathrm{TF}}(\mathbf{x})+\left(V_{\mathrm{OL}}(\mathbf{x}-\mathbf{u})-\mu\right) n_{\mathrm{TF}}(\mathbf{x})\right] \\
& =\frac{d_{z}}{2 g} \int_{\text {core }} d^{2} x\left[\mu-V_{\mathrm{OL}}(\mathbf{x}-\mathbf{u})\right]^{2} \\
& \simeq-\frac{d_{z} \mu}{g} \int_{\text {core }} d^{2} x V_{\mathrm{OL}}(\mathbf{x}-\mathbf{u})+O\left(s^{2} E_{\mathrm{R}}^{2}\right) .
\end{aligned}
$$

Performing the integral on a disk with radius $\xi$ we arrive at the same form as in Eq. (66). The only difference is the prefactor, which depends on $q \xi$,

$$
Q_{\text {core }}(q \xi)=\frac{J_{1}(2 q \xi)}{2 q \xi}
$$

Consistent with our previous remarks, this contribution of the vortex core to the position dependent energy is smaller than the kinetic energy contribution. It adds to the latter contribution given in Eq. (17) and hence we define $Q \equiv Q_{\text {kin }}+Q_{\text {core }}$. Putting things together, 
the potential energy of a vortex described by the ansatz of Eq. (44) in a two dimensional optical lattice is given by 68$]$

$$
U_{\text {pin }}(\mathbf{u})=\frac{d_{z}}{8 a_{s}} s E_{\mathrm{R}} Q(q \xi)\left[\cos \left(2 q u_{x}\right)+\cos \left(2 q u_{y}\right)\right] .
$$

It is clearly seen that the potential energy is minimal if the vortices are located at the maxima of the optical potential. This is expected, since at these maxima the condensate density, and hence the kinetic energy associated with the superfluid motion, is minimal. The expression in Eq. (11) is regarded as a pinning potential experienced by vortices in a condensate loaded in a optical lattice.

\section{B. Vortex interactions}

The interaction energy of two vortices must be known explicitly to calculate the ground state structure of vortex lattices. We calculate this interaction energy by using the following ansatz for the condensate wave function

$$
\Psi(\mathbf{x})=\sqrt{n} \Theta(R-|\mathbf{x}|) \Theta[|\mathbf{x}-\mathbf{u}| / \xi-1] \Theta[|\mathbf{x}+\mathbf{u}| / \xi-1] \exp [i \phi(\mathbf{x}, \mathbf{u})+i \phi(\mathbf{x},-\mathbf{u})]
$$

This form is a generalization of the ansatz in Eq. (4) to the case of two vortices in a disk-shaped condensate with radius $R$ and average density $n$, oppositely displaced over a distance $|\mathbf{u}|$ from the origin. The reason that we do not explicitly take into account the spatial inhomogeneity of the condensate density due to the optical lattice potential in the calculation of the vortex interaction energy, is that most of the vortices in the vortex lattice are separated by more than one optical lattice constant, such that the effect of a spatially varying density profile on the vortex interactions is averaged out. In the relevant limit where the healing length is small compared to the system size, the only significant contribution comes from the kinetic energy of the condensate. For simplicity we place the vortices along the $x$-axis, $\left(u_{x}, u_{y}\right)=\left(\frac{r}{2}, 0\right)$, which leads for the energy of the system to

$$
\begin{aligned}
V(r) & =\frac{\hbar^{2} d_{z} n}{2 M} \int_{0}^{2 \pi} d \theta \int_{0}^{R} d \rho \rho \frac{-64 \rho^{2}}{r^{4}+16 \rho^{4}-8 r^{2} \rho^{2} \cos 2 \theta} \\
& =128 \pi \frac{\hbar^{2} d_{z} n}{2 M} \int_{0}^{R} d \rho \rho \frac{\rho^{2}}{16 \rho^{4}-r^{4}} \operatorname{sgn}\left(4 \rho^{2}-r^{2}\right) .
\end{aligned}
$$

Here $\rho$ is the radial coordinate and $\theta$ is the polar angle. The effect of the condensate density

profile is incorporated by simply excluding the contribution of the vortex cores from the 
expression in Eq. (13) such that

$$
\begin{aligned}
V(r) & =\frac{64 \pi \hbar^{2} d_{z} n}{M}\left[-\int_{0}^{(r-\xi) / 2} d \rho \rho \frac{\rho^{2}}{16 \rho^{4}-r^{4}}+\int_{(r+\xi) / 2}^{R} d \rho \rho \frac{\rho^{2}}{16 \rho^{4}-r^{4}}\right] \\
& =-\frac{\pi \hbar^{2} d_{z} n}{M} \lim _{\tilde{R} \rightarrow \infty} \log \left[\frac{16 \tilde{r}^{6}-4 \tilde{r}^{4}+4 \tilde{r}^{2}-1}{\tilde{r}^{4}\left(16 \tilde{R}^{4}-\tilde{r}^{4}\right)}\right],
\end{aligned}
$$

where we defined $\tilde{R} \equiv R / \xi$ and $\tilde{r} \equiv r / \xi$ and also took the limit $\tilde{R} \rightarrow \infty$. The latter result is divergent with increasing system size. The finite, interaction energy of the two vortex

configuration is isolated by subtracting the divergent constant $\frac{\pi \hbar^{2} d_{z} n}{M} \lim _{\tilde{R} \rightarrow \infty} \log \left[1 / \tilde{R}^{4}\right]$ from the expression in Eq. (14) and evaluating the limit $\tilde{R} \rightarrow \infty$. The resulting expression does not depend on the system size and behaves like

$$
V(r)=-\frac{2 \pi \hbar^{2} d_{z} n}{M} \log \left(\frac{r}{\bar{\xi}}\right),
$$

for $r \gg \xi$. This is the well-known long range interaction potential experienced by singly quantized vortices in two dimensions [49].

In the next section we will use the results for the vortex pinning potential and the vortex interaction energy to calculate the energy of a lattice of vortices.

\section{ENERGY OF A VORTEX LATTICE IN A PERIODIC POTENTIAL}

In principle, to calculate the equilibrium positions of the vortices, we have to minimize the total energy as a function of the coordinates of the vortices. Clearly, for a large number of vortices this is unfeasible. It is known, however, that in the limit of strong pinning, the vortices form regular lattices [32, 33, 34, 35, 36]. Therefore, to find the phase diagram of the system, we minimize the energy of the system assuming that the vortices form a regular lattice. This procedure neglects the fact that for small pinning potential the hexagonal Abrikosov vortex lattice is slightly distorted by the pinning potential [36].

To carry out the above minimization procedure, it is easiest to parameterize a unit cell of the vortex lattice for a given filling factor $\nu$. The filling factor is defined as the number of vortices per pinning center, i.e., per minima of the pinning potential. In terms of the density of vortices it is equal to $\nu=n_{v} a^{2}$, where $n_{v}$ is the two-dimensional density of vortices that is set by the rotation frequency $\Omega$ as $n_{v}=M \Omega /(\pi \hbar)$ [48], and $a=\pi / q$ is the optical lattice constant. 
We consider commensurate filling factors smaller than one, i.e, $\nu=\frac{1}{k}$, with $k$ a positive integer. All possible vortex lattice unit cells corresponding to such commensurate vortex lattices at a particular filling factor can be found by factorizing $k$ in products $l \cdot m$, with $l$ and $m$ positive integers, and arranging vortices on the sides of rectangles of size $l a \times m a$, as shown in Fig. 1. Varying the vortex positions along the sides of the rectangle, keeping the area of the unit cell constant, gives all possible primitive commensurate lattice structures for the vortex lattice. For a vortex lattice of filling $\nu$ this procedure is parameterized by

$$
\mathbf{u}(\alpha, \beta ; l, m)=a\left(\begin{array}{cc}
\sqrt{1+\alpha l \beta m} & \alpha l \\
\beta m & \sqrt{1+\alpha l \beta m}
\end{array}\right)\left(\begin{array}{c}
l n_{x} \\
m n_{y}
\end{array}\right)
$$

with $n_{i} \in \mathbb{Z}$ and $0 \leq \alpha, \beta \leq \frac{1}{2}$. Notice that the transformation matrix in the above expression preserves the area of the unit cell, since its determinant equals unity. This ensures that we are considering lattice configurations with equal vortex density. The more familiar parameters of a unit cell of a two-dimensional lattice, the angle $\varphi$ between the primitive lattice vectors and the ratio of their lengths, $\kappa=L_{1} / L_{2}$, are related to $\alpha$ and $\beta$ by

$$
\begin{aligned}
& \frac{\cos \varphi}{\kappa}=\frac{m(\alpha l+\beta m) \sqrt{1+\alpha l \beta m}}{l[1+\beta m(\alpha l+\beta m)]}, \\
& \frac{\sin \varphi}{\kappa}=\frac{m}{l[1+\beta m(\alpha l+\beta m)]} .
\end{aligned}
$$

The interaction energy $E_{\text {int }}$ per unit cell as function of $\varphi$ and $\kappa$ for an infinite twodimensional lattice of vortices subject to the logarithmic interaction potential of Eq. (15) was calculated by Campbell et al. [50]. Cast in a dimensionless form their result reads

$$
\begin{aligned}
& \tilde{E}_{\text {int }} \equiv \frac{E_{\text {int }}}{\left(\pi \hbar^{2} d_{z} n / M\right)}=\frac{\pi}{6} \frac{\sin \varphi}{\kappa}-\log \left[2 \pi\left(\frac{\sin \varphi}{\kappa}\right)^{\frac{1}{2}}\right] \\
& -\log \left\{\Pi _ { j = 1 } ^ { \infty } \left[1-2 e^{-2 \pi j|\sin \varphi| / \kappa} \cos \left(2 \pi j \frac{\cos \varphi}{\kappa}\right)\right.\right. \\
& \left.\left.+e^{-4 \pi j|\sin \varphi| / \kappa}\right]\right\}
\end{aligned}
$$

It is important to realize that the interaction energy per vortex is divergent for an infinite vortex lattice, and that the above expression gives the relative interaction energy for configurations with equal vortex density. The absolute minimum of the dimensionless interaction energy in Eq. (18) corresponds to a hexagonal vortex lattice structure, i.e., the Abrikosov vortex lattice with $l=m=\sqrt{k}$ and $\alpha l=\beta m=\sqrt{1 / \sqrt{3}-1 / 2}$ or $(\varphi, \kappa)=(\pi / 3,1)$, and is equal to $\tilde{E}_{\text {int }}=-1.32112$. Note that this lattice is incommensurate with the optical lattice. 


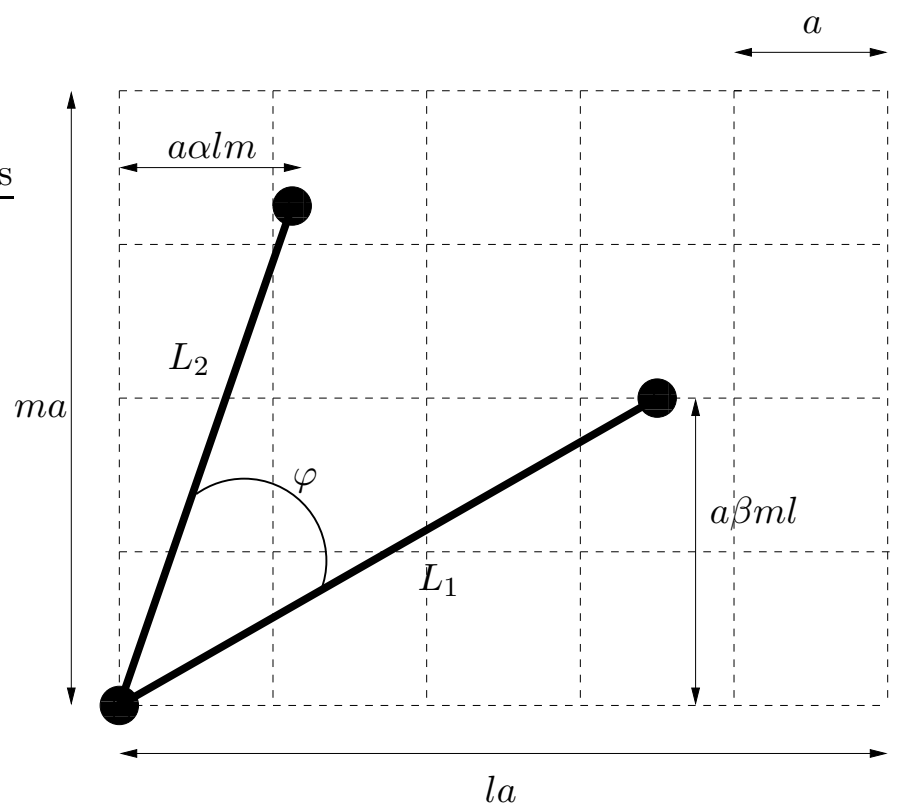

FIG. 1: Two ways to parametrize the unit cell of a vortex lattice, using parameters $(\alpha l, \beta m)$ and $(\varphi, \kappa)$, with $\kappa=L_{1} / L_{2}$. The relation between those parametrizations is given by the expressions in Eq. (17). The grid indicates the pinning potential with lattice constant $a$.

The pinning energy per unit cell is found by substituting Eq. (16) in Eq. (11), summing over all $n_{x}$ and $n_{y}$, and dividing the result by the number of unit cells,

$$
\begin{aligned}
E_{\text {pin }}(\alpha, \beta ; l, m) & =\lim _{P \rightarrow \infty} \frac{1}{4 P^{2}} \sum_{n_{x}=-P}^{P} \sum_{n_{y}=-P}^{P} U_{\text {pin }}[\mathbf{u}(\alpha, \beta ; l, m)] \\
& =-\frac{d_{z}}{8 a_{s}} s E_{\mathrm{R}} Q(q \xi)\left[\delta_{\beta m \in \mathbb{Z}}+\delta_{\alpha l \in \mathbb{Z}}\right] .
\end{aligned}
$$

This form of the pinning energy per unit cell is what we expect on an infinite lattice. Only if the vortices form a lattice that is commensurate with the optical lattice, they give a nonzero contribution to the pinning energy. This is why we consider only commensurate fillings, since we expect structural transitions at these fillings. Incommensurate vortex lattices have zero potential energy per unit cell on average. For $\nu \leq 1$ there are three possible outcomes for the pinning energy in Eq. (19). i) A phase in which all the vortices are pinned by optical lattice maxima at $E_{\mathrm{pin}}=-\frac{d_{z}}{4 a_{s}} s E_{\mathrm{R}} Q(q \xi)$, ii) a phase in which a half of the vortices is pinned at $E_{\text {pin }}=-\frac{d_{z}}{8 a_{s}} s E_{\mathrm{R}} Q(q \xi)$, and $\left.i i i\right)$ an unpinned phase at $E_{\mathrm{pin}}=0$ for any vortex lattice that is incommensurate with the optical lattice. The precise geometry of the unit cell of these vortex lattices is determined further by minimization of the interaction energy in Eq. (18). Of course, the structure of the unpinned phase is always hexagonal, corresponding to the 
global minimum of the interaction energy.

To end this section, we would like to point out that, since the interaction energy of the vortex lattice is derived by summing the expression for the interaction energy of two vortices over all pairs of vortices, we have implicitly assumed that the vortex density is so low that the vortex cores never overlap, and that we are therefore allowed to neglect three-vortex interactions, and interactions of higher order. A similar argument validates the derivation of the pinning energy of the vortex lattice by summing the single-vortex pinning potential over the number of vortices. In the numerical calculations of $\mathrm{Pu}$ et al. 47], these authors observed that for filling larger than one the vortices form pinned phases where pairs of vortices are pinned, and hence two vortices get very close together. Since our approximations break down in this case, we study only phases with a filling factor smaller than one.

\section{PHASE DIAGRAMS}

The energy per unit cell of the vortex lattice, obtained by adding the pinning energy of Eq. (19) and the interaction energy of Eq. (18), enables us to calculate the zero-temperature phase diagram of the vortex lattice structure at a certain filling. As already mentioned, we consider systems with filling factor $\nu=\frac{1}{k}$ with $k$ a nonnegative integer larger than one.

The dimensionless energy per unit cell of the vortex lattice reads

$$
\left(\frac{4 a_{s}}{\mu d_{z}}\right) E(\alpha, \beta ; l, m)=\tilde{E}_{\mathrm{int}}(\alpha, \beta ; l, m)-\frac{1}{2} \frac{s E_{\mathrm{R}}}{\mu} Q(q \xi)\left[\delta_{\beta m \in \mathbb{Z}}+\delta_{\alpha l \in \mathbb{Z}}\right],
$$

where we used that $\mu \simeq g n$. It is most convenient to minimize this expression in the plane spanned by the dimensionless parameters $q \xi$ and $\frac{s E_{\mathrm{R}}}{\mu}$. This leads to the three phases discussed in the previous section. However, the presence of the half-pinned vortex configuration depends on the filling factor, implying different phase diagrams for even and odd $k$. Since the structure of the lattice does not change continuously, the phases are separated by a first-order transition.

In the case of even $k$, the half-pinned lattice is absent, since the pinning centers are distributed such that the minimum energy configuration is always a fully-pinned lattice. The phase diagram thus contains two distinct phases: a fully-pinned vortex lattice and the hexagonal Abrikosov lattice. The geometry of the fully-pinned vortex lattice is determined 
such that the interaction energy is minimal [32, 33, 34, 35, 36].

If $k$ is an odd integer, the half-pinned lattice is present in the phase diagram if the pinning energy and the interaction energy are of the same order. However, this phase exists only if the inter-vortex distance and the optical lattice constant are comparable in size.

In Ref. [37] we discussed the case of one vortex per optical lattice unit cell, i.e., $\nu=1$. We now discuss three distinct examples in detail. The results are summarized in Fig. 2,

\section{A. $\quad \nu=\frac{1}{2}$}

For $\nu=\frac{1}{2}$ the phase diagram contains two phases and is depicted in Fig. 2 (a). For weak pinning the vortices are not pinned and form a hexagonal Abrikosov lattice. For strong pinning all vortices are located on the minimum of the pinning potential, and form a square lattice with $(\alpha, \beta)=\left(0, \frac{1}{2}\right)$ and $(l, m)=(1,2)$. Note that, as opposed to the $\nu=1$ case [37], which also has a square and pinned vortex lattice in the strong pinning regime, in this case the vortex lattice is rotated over an angle $\frac{\pi}{4}$ with respect to the optical lattice.

\section{B. $\nu=\frac{1}{4}$}

If there are four pinning centers per vortex, corresponding to $k=4$, we find for large strength of the optical lattice a fully-pinned triangular vortex lattice [69] with $(\alpha, \beta)=$ $\left(0, \frac{1}{4}\right), l=2$ and $m=2$. The interaction energy per unit cell of the vortex lattice of this configuration is $\tilde{E}_{\text {int }}=-1.31849$. At small optical lattice strength we find the hexagonal Abrikosov vortex lattice. The phase boundary is given by

$$
\left(\frac{s E_{\mathrm{R}}}{\mu}\right)_{\text {hexagonal-pinned }}=\frac{0.01057}{Q(q \xi)} .
$$

It is important to note that, contrary to the case of $\nu=\frac{1}{2}$ and $\nu=1$ [37], the geometry of the fully-pinned vortex lattice is in this case triangular. Since a fully-pinned square lattice has the same pinning energy as this triangular lattice, the interaction energy favors the latter. The phase diagram for this filling is shown in Fig. 2 (b). 

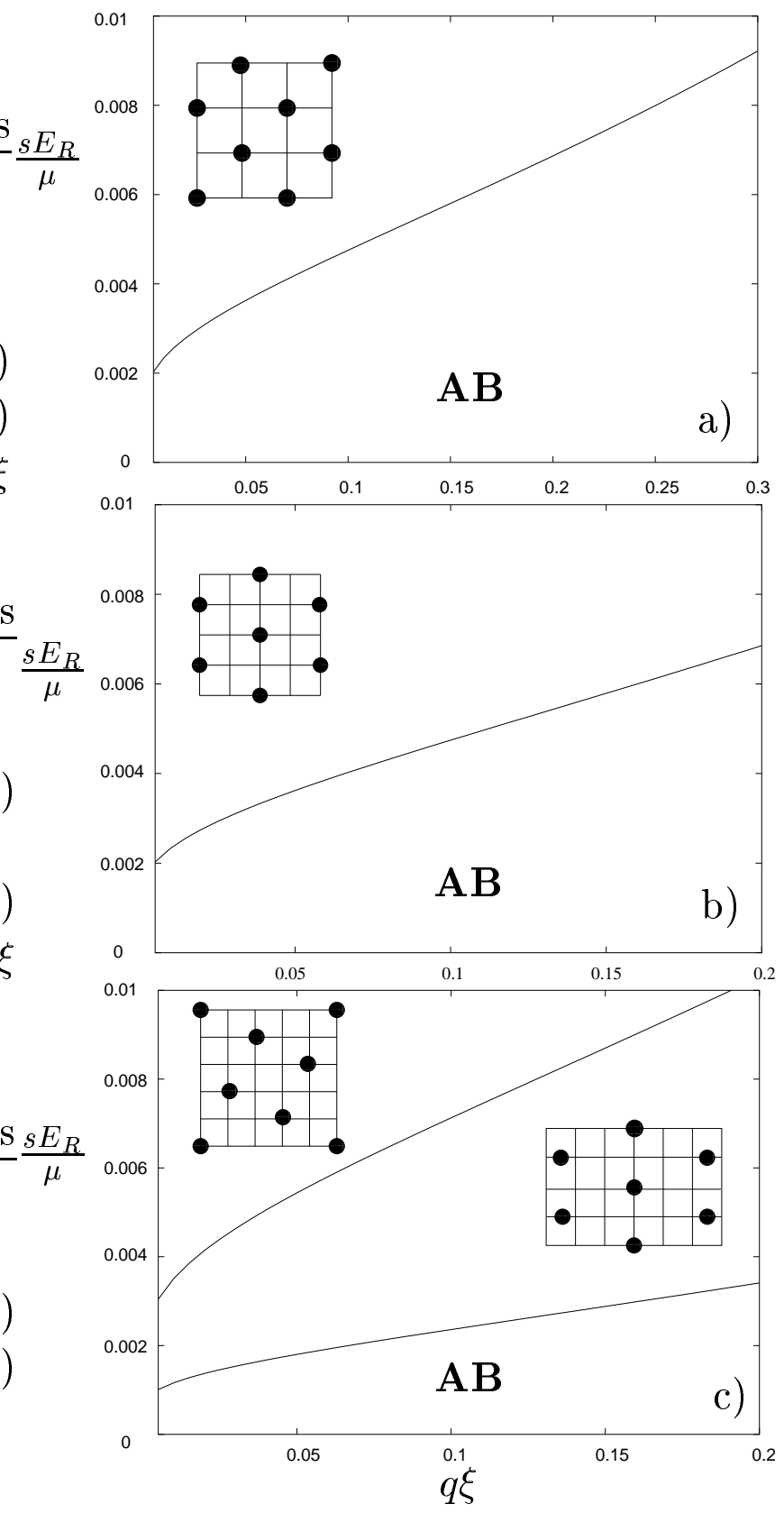

FIG. 2: Vortex phase diagram of a Bose-Einstein condensate in a two dimensional optical square lattice, for three different filling factors, a) $\nu=\frac{1}{2}$, b) $\nu=\frac{1}{4}$, and $\nu=\frac{1}{5}$. For a weak pinning potential the vortex lattice structure is always the hexagonal Abrikosov lattice (AB). The insets indicate the vortex lattice structure for stronger pinning potential. The black dots indicate the vortices, whereas the square grid indicates the pinning potential. 


\section{C. $\nu=\frac{1}{5}$}

At $k=5$ we find three phases. The result is depicted in Fig. 2 (c). First, for large strength of the optical lattice we have a fully-pinned vortex lattice with $(\alpha, \beta)=\left(\frac{2}{5}, 0\right), l=5, m=1$ and interaction energy $\tilde{E}_{\text {int }}=-1.31055$. At intermediate optical lattice strengths we find a half-pinned phase with $(\alpha, \beta)=\left(\frac{1}{2}, 0\right), l=5$ and $m=1$. The interaction energy per unit cell of this configuration equals $\tilde{E}_{\text {int }}=-1.31849$. At small lattice strength we find again the hexagonal Abrikosov vortex lattice. The boundaries between these phases are given by

$$
\left(\frac{s E_{\mathrm{R}}}{\mu}\right)_{\text {hexagonal } / \text { half-pinned }}=\frac{0.00526}{Q(q \xi)}, \quad\left(\frac{s E_{\mathrm{R}}}{\mu}\right)_{\text {half-pinned } / \text { pinned }}=\frac{0.01588}{Q(q \xi)} .
$$

Similar to the $\nu=\frac{1}{2}$ case. we find that the fully-pinned vortex lattice has a square geometry, and is now rotated over an angle $\tan ^{-1}\left(\frac{1}{2}\right)$ with respect to the optical lattice. Generally, if the fully-pinned vortex lattice has a square geometry, then for filling factor $\nu=\frac{1}{k_{1}^{2}+k_{2}^{2}}$, with

$k_{1}$ and $k_{2}$ integer, the fully-pinned vortex lattice will be rotated over an angle $\tan ^{-1}\left(\frac{k_{2}}{k_{1}}\right)$ with respect to the optical lattice.

Contrary to the above mentioned filling factors, but similar to the $\nu=1$ case [37], there is an intermediate triangular vortex lattice, where half of the vortices is pinned.

\section{PINNING OF VORTICES IN TWO-COMPONENT CONDENSATES}

In this section we study the influence of a two-dimensional optical potential on vortex lattices in a mixture of Bose-Einstein condensates of two different species. Our results also apply to a Bose-Einstein condensate that consists of two hyperfine components, provided the number of atoms in each component is conserved. Along the lines of Sec. III III and IV] we calculate the ground state phase diagram for two coupled condensates each containing a vortex lattice at filling $\nu_{i}=1$ with the optical potential. Note that the fact that we take the filling factor to be the same in both species implies that the masses of both species are approximately equal.

A system of two coupled Bose-Einstein condensates is described by the following hamiltonian

$$
H=\sum_{i=1,2} \int \mathrm{d} \mathbf{x} \Psi_{i}^{*}\left[\frac{-\hbar^{2} \nabla^{2}}{2 M_{i}}+V_{\mathrm{OL}}(\mathbf{x})-\mu_{i}\right] \Psi_{i}
$$




$$
+\int \mathrm{d} \mathbf{x}\left[\frac{1}{2} g_{1}\left|\Psi_{1}\right|^{4}+\frac{1}{2} g_{2}\left|\Psi_{2}\right|^{4}+g_{12}\left|\Psi_{1}\right|^{2}\left|\Psi_{2}\right|^{2}\right]
$$

with $g_{i}=4 \pi \hbar^{2} a_{i} / M_{i}$ and $g_{12}=2 \pi \hbar^{2} a_{12} / M_{i j}$. Here $a_{12}$ is the scattering length between unlike species and the reduced mass is given by $M_{i j}=M_{i} M_{j} /\left(M_{i}+M_{j}\right)$.

In the absence of the optical potential, Mueller and Ho [51] and Kasamatsu et al. [52] theoretically predicted smooth transitions between hexagonal lattices in both components at small (intra species) interactions and interlaced square vortex lattices at larger interaction. These square lattices where observed very recently by Schweikhard et al. [53]. However, the above-mentioned transition is caused by the fact that the interaction energy is minimized if the overall density is as smooth as possible. Since we take a step function for the density profile of the vortex, this density effect is not included in our calculations. Therefore our results only make sense in the regime where this density effect is dominated by the optical potential, i.e., in the strong-pinning limit. To ensure this, the requirement $\left(\frac{s E_{R}}{\mu_{i}}\right) Q\left(q \xi_{i}\right) \gg$ $\frac{g_{12}}{g_{i}}$ must be satisfied. This implies that we must have that $g_{i} \gg g_{12}$, since we assumed that $s E_{R}<\mu_{i}$. It must be stressed that this is quite restricting as at the present day there is no experimental atomic system known which meet these requirements. However, one might expect that near an interspecies Feshbach resonance this regime of parameters is realizable. Therefore, we study the fully-pinned and half-pinned lattices and the phase transition between them.

A non-rotating two-component condensate phase separates if $g_{12}>\sqrt{g_{1} g_{2}}$ [54, 55]. The condensates mutually exclude each other, even in the absence of external potentials [56] or with rotation [52]. The vortex ground state in the latter case will not be given by a regular lattice, in general. In our calculations we restrict ourself to the regime where the system does not phase separate. In this regime, the coupling parameters satisfy $g_{i}>0$ and $g_{1} g_{2}>g_{12}^{2}$, which falls safely within the approximation discussed above. We define the dimensionless parameter $\chi^{2} \equiv g_{12}^{2} / g_{1} g_{2}$, for which these criteria imply $0<\chi^{2}<1$.

Solving the coupled equations for the condensate wave functions, derived from the hamiltonian of Eq. (23) in the Thomas-Fermi approximation, leads to the following density profile in component $i$

$$
n_{\mathrm{TF}}^{i}(\mathbf{r})=\left|\Psi_{i}\right|^{2}=\frac{1}{g_{i}} \frac{\left[\mu_{i}-V_{\mathrm{OL}}(\mathbf{r})\right]}{1-\chi^{2}}+\frac{1}{g_{12}} \frac{\left[\mu_{j}-V_{\mathrm{OL}}(\mathbf{r})\right]}{1-\frac{1}{\chi^{2}}}, \quad j \neq i
$$

We use the variational ansatz for the condensate wave function containing a vortex in com- 
ponent $i$

$$
\Psi_{i}(\mathbf{x})=\sqrt{n_{\mathrm{TF}}^{i}(\mathbf{x})} \Theta\left[|\mathbf{x}-\mathbf{u}| / \xi_{i}-1\right] \exp \left[i \phi_{i}(\mathbf{x}, \mathbf{u})\right]
$$

with $\xi_{i}=1 / \sqrt{8 \pi a_{i} n_{i}}$ and $n_{i}=\left(\mu_{i}-s E_{\mathrm{R}}\right) / g_{i}$. Furthermore, we assume that vortices in different components do not interact. As explained before, in particular we neglect the effect of the density profile caused by a vortex in one component on the vortices in the other component, which, in the absence of an optical potential, leads to the structural transitions discussed by Mueller and Ho [51] and Kasamatsu et al. [52]. Within each component, the vortex interactions are logarithmic, as derived in Sec. ЏB.

The pinning potential which is experienced by the vortex can be calculated along the lines of Sec. ПA. The first contribution, coming from the kinetic energy term of the hamiltonian in Eq. (23), is equal to

$$
U_{\text {kin }}^{i}(\mathbf{u})=\frac{d_{z}}{8 a_{i}} s E_{\mathrm{R}} Q_{\mathrm{kin}}\left(q \xi_{i}\right) G_{i}\left(g_{1}, g_{2}, g_{12}\right)\left[\cos \left(2 q u_{x}\right)+\cos \left(2 q u_{y}\right)\right],
$$

with $Q_{\text {kin }}$ given by Eq. (17). The difference with the single component case is the appearance of the factor

$$
G_{i}\left(g_{1}, g_{2}, g_{12}\right)=\frac{g_{12}-g_{i} \chi^{2}}{g_{12}\left(1-\chi^{2}\right)} .
$$

This factor is completely dependent on the various interaction strengths. The other significant (position-dependent) contribution, coming from the vortex core, involves more work,

$$
\begin{aligned}
U_{\text {core }}^{i}(\mathbf{u})= & -d_{z} \int_{\text {core }} \mathrm{d}^{2} x\left[\frac{1}{2} g_{i}\left|\Psi_{i}\right|^{4}+\left(V_{\mathrm{OL}}-\mu_{i}\right)\left|\Psi_{i}\right|^{2}+\frac{1}{2} g_{12}\left|\Psi_{1}\right|^{2}\left|\Psi_{2}\right|^{2}\right] \\
= & -d_{z} \int_{\text {core }} \mathrm{d}^{2} x\left\{\frac { g _ { i } } { 2 } \left[\frac{\left(\mu_{i}-V_{\mathrm{OL}}\right)^{2}}{g_{i}^{2}\left(1-\chi^{2}\right)^{2}}+\frac{\left(\mu_{j}-V_{\mathrm{OL}}\right)^{2}}{g_{12}^{2}\left(1-1 / \chi^{2}\right)^{2}}\right.\right. \\
+ & \left.+2 \frac{\left(\mu_{i}-V_{\mathrm{OL}}\right)\left(\mu_{j}-V_{\mathrm{OL}}\right)}{g_{i} g_{12}\left(1-\chi^{2}\right)\left(1-1 / \chi^{2}\right)}\right] \\
+ & \left.\left.\times \frac{\left(\mu_{i}-V_{\mathrm{OL}}\right)}{g_{i}\left(1-\chi^{2}\right)}+\frac{\left(\mu_{j}-V_{\mathrm{OL}}\right)}{g_{12}\left(1-1 / \chi^{2}\right)}\right]\right\} \\
= & -\frac{d_{z}}{g_{i}} \frac{1}{\chi^{2}-1}\left[\frac{\left(\mu_{j}-V_{\mathrm{OL}}\right)}{g_{j}\left(1-\chi^{2}\right)}+\frac{\left(\mu_{\mathrm{OL}}\right)}{g_{12}\left(1-1 / \chi^{2}\right)}\right] \\
\left.2 g_{j}+\mu_{j}\right) & \left.-\mu_{i}\right] \int_{\text {core }} \mathrm{d}^{2} x V_{\mathrm{OL}}(\mathbf{x}-\mathbf{u})+\mathrm{O}\left(s^{2} E_{R}^{2}\right) .
\end{aligned}
$$

If we assume that $\mu_{i} \approx \mu_{j}$ we find a contribution similar to Eq. (26). The prefactor in this case is given by $Q_{\text {core }}(q \xi)$ which is defined in Eq. (10). The total pinning potential 
experienced by a vortex in component $i$ due to the optical lattice is equal to

$$
U_{\text {pin }}^{i}(\mathbf{u})=\frac{d_{z}}{8 a_{i}} s E_{\mathrm{R}}\left[Q_{\text {kin }}\left(q \xi_{i}\right)+Q_{\text {core }}\left(q \xi_{i}\right)\right] G_{i}\left(g_{1}, g_{2}, g_{12}\right)\left[\cos \left(2 q u_{x}\right)+\cos \left(2 q u_{y}\right)\right] .
$$

This energy is dependent on the ratio of the coupling parameters. The pinning energy is minimized on lattice maxima in both components in the regime where it is the dominant energy scale. Therefore, vortices in both components tend to be on the same position.

In order to find the vortex phase diagram we minimize the interaction and pinning energy in each component, as in Sec. [IV] We assume (again) $\mu_{i} \approx \mu_{j}=\mu$. This implies $\xi_{i} \approx \xi_{j}=$ $\xi$. As mentioned before, we are only interested in lattice types which are fully-pinned or half-pinned. The phase boundary between the fully-pinned and half-pinned vortex lattices (commensurable with the optical lattice) in each component is given by

$$
\left(\frac{s E_{\mathrm{R}}}{\mu}\right)_{i}=\frac{0.01494}{Q(q \xi) G_{i}\left(g_{1}, g_{2}, g_{12}\right)} .
$$

To find all possible lattice types in the two-component system it is most convenient to parametrize the coupling parameters by $g_{1}=\kappa g_{12} \sin (\gamma)$ and $g_{2}=\kappa g_{12} \cos (\gamma)$. By varying $\gamma$ one scans along an circle segment in the $\left(g_{1}, g_{2}\right)$-plane. The non-phase separated regime in terms of the new parameters is given by $\kappa>\sqrt{2}$ and $\gamma_{-}<\gamma<\gamma_{+}$with

$$
\gamma_{ \pm}=\arccos \left[\sqrt{\frac{1}{2}\left(1 \pm \frac{\sqrt{\kappa^{4}-4}}{\kappa^{2}}\right)}\right] .
$$

We find that the phase diagram contains four different vortex lattices. In Fig. (3) the phase diagram and the lattice geometry are displayed for $q \xi=0.05$ and $\kappa=100$. Notice that the two-component phase diagram has a straightforward interpretation in terms of coexistence of phases found in the single-component case. For strong pinning the vortex lattices are both fully pinned. For the filling under consideration $(\nu=1)$ the vortices in both components therefore form a square lattice. Depending on the relative strength of the interaction, determined by the parameter $\gamma$, the vortex lattice in one of the components will change first to the half-pinned triangular geometry, as one lowers the strength of the optical potential. For sufficiently weak pinning potential both vortex lattices assume this structure.

\section{COLLECTIVE MODES}

In this section we calculate the dispersion of the collective modes of the pinned vortex lattices. In principle, this requires the calculation of the energy of the system for small 


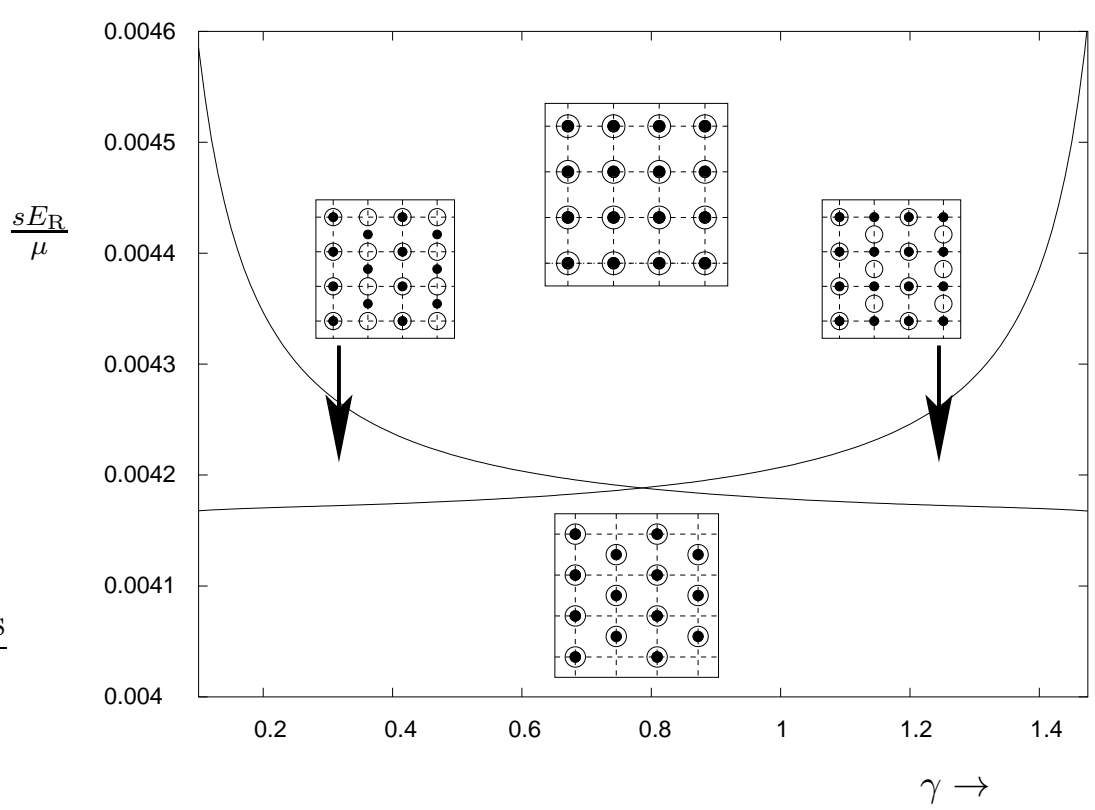

FIG. 3: Vortex phase diagram of a rotating two-component condensate in the presence of an optical lattice, at commensurate filling, $\nu_{i}=1$. The phase diagram is plotted as function of the dimensionless parameter $\gamma=\arctan \left(g_{1} / g_{2}\right)$. Black dots and circles represent vortices in different components. The dashed lines represent the pinning potential. We took $\kappa=100$ and $q \xi=0.05$ which implies $Q(0.05) \approx 3.612$.

displacements $\mathbf{u}\left(\mathbf{r}_{i}\right)$ of the vortices from their equilibrium positions $\mathbf{r}_{i} \equiv r_{i x} \hat{x}+r_{i y} \hat{y}$. In first instance, the energy of the system is then given by

$$
\begin{aligned}
E[\mathbf{u}] & =\frac{d_{z} s E_{\mathrm{R}} Q(q \xi)}{8 a_{s}} \sum_{i}\left\{\cos \left[2 q\left(r_{i x}+u_{x}\left(\mathbf{r}_{i}\right)\right)\right]+\cos \left[2 q\left(r_{i y}+u_{y}\left(\mathbf{r}_{i}\right)\right)\right]\right\} \\
& -\frac{\pi \hbar^{2} d_{z} n}{M} \sum_{i \neq j} \log \left|\frac{\mathbf{r}_{i}+\mathbf{u}\left(\mathbf{r}_{i}\right)-\mathbf{r}_{j}-\mathbf{u}\left(\mathbf{r}_{j}\right)}{\xi}\right|
\end{aligned}
$$

We perform a Fourier transform

$$
\mathbf{u}\left(\mathbf{r}_{i}\right)=\frac{1}{\sqrt{N_{x} N_{y}}} \sum_{\mathbf{k}} \mathbf{u}(\mathbf{k}) e^{i \mathbf{k} \cdot \mathbf{r}_{i}}
$$

where $N_{\alpha}$ is the number of vortices along the $\alpha$ direction of the vortex lattice, and the momentum sum is restricted over values $k_{\alpha}=2 \pi n_{\alpha} / L_{\alpha}$ in the first Brillouin zone, where $n_{\alpha}$ is an integer and $L_{\alpha}$ is the size of the vortex lattice in the $\alpha$ direction. Note that throughout this section we use Greek symbols to indicate two-dimensional Cartesian components, i.e., $\alpha, \beta \in\{x, y\}$. We also sum over repeated Greek indices. 
We expect that after this Fourier transformation the energy of the vortex lattice for small displacements will, up to an irrelevant constant, be given by

$$
E[\mathbf{u}]=\frac{1}{2} \sum_{\mathbf{k}} D_{\alpha \beta}(\mathbf{k}) u_{\alpha}(-\mathbf{k}) u_{\beta}(\mathbf{k}),
$$

where the so-called dynamical matrix is the sum of three contributions

$$
D_{\alpha \beta}(\mathbf{k})=D_{\alpha \beta}^{\mathrm{EL}}(\mathbf{k})+D_{\alpha \beta}^{\mathrm{LR}}(\mathbf{k})+D_{\alpha \beta}^{\mathrm{OL}}(\mathbf{k})
$$

The first contribution $D_{\alpha \beta}^{\mathrm{EL}}(\mathbf{k})$ takes into account the interaction between neighboring vortices and follows from elasticity theory. Although the elastic constants that will enter in the expression for $D_{\alpha \beta}^{\mathrm{EL}}(\mathbf{k})$ can in principle be calculated from the interaction energy in Eq. (32), such a calculation is beyond the scope of this paper, and we will adopt a more phenomenological point of view and write down the most general form for $D_{\alpha \beta}^{\mathrm{EL}}(\mathbf{k})$ allowed by symmetry arguments, for each lattice under consideration [57]. The second contribution

$$
D_{\alpha \beta}^{\mathrm{LR}}(\mathbf{k})=\frac{8 \pi^{2} \hbar^{2} d_{z} n}{A M} \frac{k_{\alpha} k_{\beta}}{k^{2}+\lambda^{-2}}
$$

where $A$ is the area of the unit cell of the vortex lattice, is independent of the structure of the lattice and follows from the long-range nature of the logarithmic interactions, which has to be taken into account separately [58, 59, 60]. Note that we have explicitly included a finite range $\lambda$ of the logarithmic interactions, to ensure that $D_{\alpha \beta}^{\mathrm{LR}} \rightarrow 0$ as $k \rightarrow 0$. After taking the long wavelength limit, we can safely take $\lambda \rightarrow 0$. The final contribution $D_{\alpha \beta}^{\mathrm{OL}}(\mathbf{k})$ is due to the optical lattice.

The dispersion of the collective modes is determined by putting the determinant of the matrix

$$
M_{\alpha \beta}(\mathbf{k}, \omega)=D_{\alpha \beta}(\mathbf{k})-\epsilon_{\alpha \beta} 4 \pi i n d_{z} \hbar \omega
$$

equal to zero. Here, $\epsilon_{\alpha \beta}$ is the antisymmetric Levi-Cevita tensor in two-dimensions, that takes into account the Euler dynamics of the vortices [48, 49].

We will now calculate the dynamical matrix for each type of vortex lattice considered in the previous section, i.e, for the hexagonal, half-pinned, and fully-pinned vortex lattice, in the long-wavelength limit, and use these results to calculate the phonon spectrum of the vortex lattice. 


\section{A. Hexagonal vortex lattice}

For a hexagonal vortex lattice we have that [57]

$$
D_{\alpha \beta}^{\mathrm{EL}}(\mathbf{k})=K_{H} k_{\alpha} k_{\beta}+\mu_{H} \delta_{\alpha \beta} k^{2}
$$

where $K_{H}$ is the bulk modulus, and $\mu_{H}$ is the shear modulus of the hexagonal vortex lattice. Using this result together with Eq. (36), we find for the frequency of the collective modes in the absence of an optical lattice that

$$
\hbar \omega_{\mathbf{k}}=\frac{k}{4 \pi n d_{z}} \sqrt{\mu_{H}\left[\frac{8 \pi^{2} \hbar^{2} d_{z} n}{a_{v}^{2} M}+\left(K_{H}+\mu_{H}\right) k^{2}\right]} .
$$

For large wavelengths we have that $\hbar \omega_{\mathbf{k}} \simeq c_{T} k$, where the sound velocity of the so-called Tkachenko waves is given by

$$
c_{T}=\frac{1}{4 \pi n d_{z}} \sqrt{\frac{8 \pi^{2} \mu_{H} \hbar^{2} d_{z} n}{a_{v}^{2} M}} .
$$

We could now fix the value for the shear modulus of the vortex lattice $\mu_{H}$ by demanding that the Tkachenko sound velocity is equal to $\sqrt{\hbar \Omega /(4 M)}$, the result known from the hydrodynamic theory of a vortex lattice [9, 61]. This would, however, not be consistent, since the value for $\mu_{H}$ should follow from the expression for the energy of the vortex lattice in Eq. (32), and may lead to a different sound velocity due to the variational approximations we have made in the description of the vortex lattice. Note that is crucial to take into account the long-range interactions of the vortices by means of the dynamical matrix in Eq. (36) to get a linear dispersion at long wavelengths, since an omission of this part in the dynamical matrix would lead to a quadratic dispersion. Moreover, we would like to point out that due to the fact that vortices are described by Euler dynamics we find only one mode, instead of two modes for the case of a lattice of particles that obey Newtonian dynamics.

The polarization of the vortex lattice phonons is determined by the eigenvector of the matrix in Eq. (37), corresponding to the eigenfrequency in Eq. (39). Generally, the displacements are given by $\mathbf{u}\left(\mathbf{r}_{i}, t\right)=\mathbf{u}_{\mathbf{k}, 0} e^{i \mathbf{k} \cdot \mathbf{r}_{i}-i \omega_{\mathbf{k}} t}$, where $\mathbf{u}_{\mathbf{k}, 0}$ is the eigenvector. For a wave in the $y$-direction we have that

$$
\mathbf{u}_{\mathbf{k}, 0} \propto\left(\frac{i\left[\frac{8 \pi^{2} \hbar^{2} d_{z} n}{a_{v}^{2} M}+k_{y}^{2}\right]}{k_{y} \sqrt{\mu_{H}\left[\frac{8 \pi^{2} \hbar^{2} d_{z} n}{a_{v}^{2} M}+\left(K_{H}+\mu_{H}\right) k^{2}\right]}}\right) \approx\left(\begin{array}{c}
\frac{i}{\mu_{H} k_{y}} \sqrt{\frac{8 \pi^{2} \mu_{H} \hbar^{2} d_{z} n}{a_{v}^{2} M}} \\
1
\end{array}\right),
$$


which shows that the vortices move on an ellipse with the long axis perpendicular to the direction of propagation. In the limit $\mathbf{k} \rightarrow 0$, the wave is almost transverse.

The translation symmetry of the system is broken explicitly in the presence of an optical lattice. The collective modes therefore acquire a gap, i.e., there is a minimum amount of energy required to excite a phonon. Considering the part of the energy of Eq. (32) which corresponds to the pinning energy of the vortices and expanding it, we have, up to an irrelevant constant,

$$
\begin{aligned}
E^{\mathrm{OL}}[\mathbf{u}]=-2 q^{2} \frac{d_{z} s E_{\mathrm{R}} Q(q \xi)}{8 a_{s}} \sum_{\mathbf{k}, \mathbf{k}^{\prime}} & \left\{u_{x}(\mathbf{k}) u_{x}\left(\mathbf{k}^{\prime}\right) \sum_{j} \cos \left[2 q r_{j x}\right] e^{i\left(\mathbf{k}+\mathbf{k}^{\prime}\right) \cdot \mathbf{r}_{j}}\right. \\
+ & \left.u_{y}(\mathbf{k}) u_{y}\left(\mathbf{k}^{\prime}\right) \sum_{j} \cos \left[2 q r_{j y}\right] e^{i\left(\mathbf{k}+\mathbf{k}^{\prime}\right) \cdot \mathbf{r}_{j}}\right\},
\end{aligned}
$$

with the positions of vortices in the hexagonal lattice given by

$$
\mathbf{r}_{i}=a_{v} \sqrt{\frac{2}{\sqrt{3}}}\left[\left(i_{x}+\frac{1}{2} i_{y}\right) \hat{x}+\frac{1}{2} \sqrt{3} i_{y} \hat{y}\right] .
$$

Summing over these lattice coordinates in Eq. (42) gives zero. This is in contradiction with the fact, that on symmetry grounds we expect a finite gap for the collective modes. To overcome this problem we must take into account that the real unpinned vortex ground state in a weak periodic pinning potential is a slightly distorted hexagonal lattice [36]. This comes about because the periodic potential exerts a small net force on the vortices arranged in the hexagonal lattice. The small distortion of the vortex positions is a modulation around the equilibrium of the hexagonal lattice $\mathbf{r}_{i} \mapsto \mathbf{r}_{i}+\mathbf{R}\left(\mathbf{r}_{i}\right)$. Following Pogosov et al. [36] we find for a square two-dimensional periodic potential

$$
R_{\alpha}\left(\mathbf{r}_{i}\right)=\frac{s E_{\mathrm{R}}}{2 q \mu_{H} A} \frac{d_{z}}{8 a_{s}} Q(q \xi) \sin \left[2 q r_{i \alpha}\right]
$$

The modulation of the vortex coordinates around the positions of the regular hexagonal lattice involves a factor $\eta=\frac{s E_{\mathrm{R}}}{\mu_{H} A}$. To keep the displacements small, the condition $\eta \ll 1$ must be satisfied.

Summing over the displaced hexagonal vortex lattice in Eq. (42) indeed leads to a nonzero energy gap. Numerical evaluation of Eq. (42) on a large lattice gives a contribution to the dynamical matrix which is roughly linear in $\eta$,

$$
D_{\alpha \beta}^{\mathrm{OL}} \approx 0.5 \eta s E_{\mathrm{R}}\left[\frac{q d_{z} Q(q \xi)}{4 a_{s}}\right]^{2} \delta_{\alpha \beta}, \quad \eta \ll 1 .
$$


Including this in the calculation of the collective modes, we find

$$
\begin{aligned}
\hbar \omega_{\mathbf{k}}= & \frac{1}{4 \pi n d_{z}}\left[0.5 \eta s E_{\mathrm{R}}\left(\frac{q d_{z} Q(q \xi)}{4 a_{s}}\right)^{2}+\mu_{H} k^{2}\right]^{\frac{1}{2}} \times \\
& {\left[\frac{8 \pi^{2} \hbar^{2} d_{z} n}{a_{v}^{2} M}+0.5 \eta s E_{\mathrm{R}}\left(\frac{q d_{z} Q(q \xi)}{4 a_{s}}\right)^{2}+\left(K_{H}+\mu_{H}\right) k^{2}\right]^{\frac{1}{2}} . }
\end{aligned}
$$

The gap takes the form

$$
\hbar \omega_{\mathbf{0}} \approx \frac{0.5 \eta s E_{\mathrm{R}}}{4 \pi n d_{z}}\left[\frac{q d_{z} Q(q \xi)}{4 a_{s}}\right]^{2}
$$

\section{B. Half-pinned vortex lattice}

In the case of the half-pinned vortex lattice, which is always triangular, the dynamical matrix that follows from elasticity theory is given by [57]

$$
D_{\alpha \beta}^{\mathrm{EL}}(\mathbf{k})=K_{T} k_{\alpha} k_{\beta}+\mu_{T} \delta_{\alpha \beta} k^{2}+\kappa_{T}\left(1-\delta_{\alpha \beta}\right) k_{\alpha} k_{\beta}+\delta \mu_{T} \tau_{\alpha \beta}^{z} k_{\alpha} k_{\beta}
$$

where $K_{T}$ is the bulk modulus of the triangular vortex lattice, and $\kappa_{T}$ and $\mu_{T}$ denote the Lamé constants. For a square lattice we have that the parameter $\delta \mu_{T}$ is equal to zero, as we will see below. In the above expression $\tau_{\alpha \beta}^{z}$ denotes the Pauli matrix.

To find the contribution due to the optical lattice, we consider specifically the half-pinned case at filling $\nu=\frac{1}{5}$, as shown in Fig. 2. We parametrize the equilibrium position of the vortices as

$$
\mathbf{r}_{i}=\frac{5}{2} i_{x} a \hat{x}+2\left[\frac{1-(-1)^{i_{x}}}{4}\right] a \hat{y}+2 i_{y} a \hat{y} .
$$

With the use of this parametrization we find that the energy of the vortex lattice due to the

pinning of the optical lattice is, for small displacements $\mathbf{u}\left(\mathbf{r}_{i}\right)$ from the equilibrium positions, given by

$$
\begin{aligned}
E^{\mathrm{OL}}[\mathbf{u}] & =\frac{d_{z} s E_{\mathrm{R}} Q(q \xi)}{8 a_{s}} \sum_{i}\left\{\cos \left[2 q\left(r_{i x}+u_{x}\left(\mathbf{r}_{i}\right)\right)+\pi\right]+\cos \left[2 q\left(r_{i y}+u_{y}\left(\mathbf{r}_{i}\right)\right)+\pi\right]\right\} \\
& \simeq \frac{d_{z} s E_{\mathrm{R}} q^{2} Q(q \xi)}{4 a_{s}}\left[\sum_{i}(-1)^{i_{x}+1} u_{x}^{2}\left(\mathbf{r}_{i}\right)+u_{y}^{2}\left(\mathbf{r}_{i}\right)\right],
\end{aligned}
$$

where we have omitted an irrelevant constant. Note that we have translated the optical lattice potential to ensure that there is a vortex at the origin, consistent with the parametrization in Eq. (49). Using that

$$
\sum_{i} e^{i \mathbf{k} \cdot \mathbf{r}_{i}}=N_{x} N_{y} \delta_{\mathbf{k}, \mathbf{0}}
$$


we find after a Fourier transform in first instance that

$$
E^{\mathrm{OL}}[\mathbf{u}]=\frac{d_{z} s E_{\mathrm{R}} q^{2} Q(q \xi)}{4 a_{s}} \sum_{\mathbf{k}}\left[\sum_{i} \sum_{\mathbf{k}^{\prime}}(-1)^{i_{x}} e^{i\left(\mathbf{k}+\mathbf{k}^{\prime}\right) \cdot \mathbf{r}_{i}} u_{x}(\mathbf{k}) u_{x}\left(\mathbf{k}^{\prime}\right)+u_{y}(\mathbf{k}) u_{y}(-\mathbf{k})\right] .
$$

The sum $i_{x}$ in the second term of this equation is evaluated by splitting it into a sum over $i_{x}$ even and $i_{x}$ odd. If we denote $\mathbf{k}+\mathbf{k}^{\prime}=\left(2 \pi n_{x} / L_{x}, 2 \pi n_{y} / L_{y}\right)$, we have that

$$
\begin{aligned}
& \sum_{i}(-1)^{i_{x}+1} e^{i\left(\mathbf{k}+\mathbf{k}^{\prime}\right) \cdot \mathbf{r}_{i}}=-\sum_{i_{x}, i_{y}}(-1)^{i_{x}} e^{2 \pi i n_{x} i_{x} / N_{x}+2 \pi i n_{y} / N_{y}\left\{\left[\frac{1-(-1)^{i_{x}}}{4}+i_{y}\right]\right\}} \\
& =-N_{y} \delta_{n_{y}, 0}\left[\sum_{p} e^{2 \pi i n_{x}(2 p) / N_{x}}-\sum_{l} e^{2 \pi i n_{x}(2 p+1) / N_{x}-\pi i n_{y} / N_{y}}\right] \\
& =-N_{x} \delta_{n_{x}, 0} N_{y} \delta_{n_{y}, 0}\left[\frac{1}{2}-\frac{1}{2} e^{2 \pi i n_{x} / N_{x}-\pi i n_{y} / N_{y}}\right] .
\end{aligned}
$$

With the use of this result we have that

$$
E^{\mathrm{OL}}[\mathbf{u}]=\frac{d_{z} s E_{\mathrm{R}} q^{2} Q(q \xi)}{4 a_{s}} \sum_{\mathbf{k}} u_{y}(\mathbf{k}) u_{y}(-\mathbf{k}) \equiv \frac{1}{2} \sum_{\mathbf{k}} D_{\alpha \beta}^{\mathrm{OL}}(\mathbf{k}) u_{\alpha}(\mathbf{k}) u_{\beta}(-\mathbf{k})
$$

so that the contribution to the dynamical matrix due to the optical lattice is given by 70 ]

$$
D_{\alpha \beta}^{\mathrm{OL}}(\mathbf{k})=\frac{d_{z} s E_{\mathrm{R}} q^{2} Q(q \xi)}{2 a_{s}} \delta_{\alpha, y} \delta_{\alpha \beta}
$$

With the above results, we find that the collective mode dispersion is given by

$$
\begin{aligned}
\left(4 \pi n d_{z} \hbar \omega_{\mathbf{k}}\right)^{2} & =\mu_{T}^{2} k^{4}+\mu_{T}^{2} k^{2}\left(K_{T} k^{2}+\frac{8 \pi^{2} \hbar^{2} d_{z} n}{a_{v}^{2} M}\right)-\kappa_{T}\left[\frac{16 \pi^{2} \hbar^{2} d_{z} n}{a_{v}^{2} M}+\left(2 K_{T}+\kappa_{T}\right) k^{2}\right] \frac{k_{x}^{2} k_{y}^{2}}{k^{2}} \\
& -\frac{1}{4} \delta \mu_{T}^{2} k_{x}^{2} k_{y}^{2}+\mu_{T} \delta \mu_{T} k^{2}\left(k_{x}^{2}-k_{y}^{2}\right) \\
& +\frac{d_{z} s E_{\mathrm{R}} q^{2} Q(q \xi)}{2 a_{s}}\left[k_{x}^{2}\left(K_{T}+\mu_{T}-\frac{\delta \mu_{T}}{2}+\frac{8 \pi^{2} \hbar^{2} d_{z} n}{a_{v}^{2} M k^{2}}\right)+\mu_{T} k_{y}^{2}\right]
\end{aligned}
$$

Interestingly, this dispersion is gapless, i.e., $\hbar \omega_{\mathbf{0}}=0$. The eigenvector corresponding to this eigenfrequency is given by $(1,0)$, and so the displacement of the vortices is along the $x$-axis. Physically, this is understood because it does not cost any energy to uniformly displace all the vortices in the $x$-direction when the vortices are forming a half-pinned lattice with the geometry shown in Fig. 2] (See again [69] and [0].) This comes about because under a uniform translation of the vortices in the $x$-direction, half of the vortices move away from an energy minimum and therefore increase their energy, the other half moves downhill from an energy saddle-point, precisely compensating this increase. 


\section{Fully-pinned vortex lattice}

The structure of the fully pinned vortex lattice at certain filling is triangular, in general. Therefore, the contribution to the dynamical matrix due to the elasticity of the vortex lattice takes the form of Eq. (48) with elastic constants $K_{P}, \mu_{P}, \kappa_{P}$ and $\delta \mu_{P}$. In the special cases for which the fully pinned vortex lattice has a square structure, we have that $\delta \mu_{P}=0$. Since all the vortices are positioned at the minimum of the pinning potential, the contribution of the optical lattice to the dynamical matrix is constant and diagonal, and given by

$$
D_{\alpha \beta}^{\mathrm{OL}}(\mathbf{k})=\frac{d_{z} s E_{\mathrm{R}} q^{2} Q(q \xi)}{2 a_{s}} \delta_{\alpha \beta} .
$$

Hence, we find for the phonon dispersion

$$
\begin{aligned}
\left(4 \pi n d_{z} \hbar \omega_{\mathbf{k}}\right)^{2}= & \left(\mu_{P} k^{2}+\frac{d_{z} s E_{\mathrm{R}} q^{2} Q(q \xi)}{2 a_{s}}\right)^{2} \\
& +\left(\mu_{P} k^{2}+\frac{d_{z} s E_{\mathrm{R}} q^{2} Q(q \xi)}{2 a_{s}}\right)\left[K_{P} k^{2}+\frac{8 \pi^{2} \hbar^{2} d_{z} n}{a_{v}^{2} M k^{2}}+\frac{\delta \mu_{P}}{2}\left(k_{x}^{2}-k_{y}^{2}\right)\right] \\
& +\left[\frac{\delta \mu_{P}^{2}}{4}-\kappa_{P}^{2}-2 \kappa_{P}\left(K_{P}+\frac{8 \pi^{2} \hbar^{2} d_{z} n}{a_{v}^{2} M k^{2}}\right)\right] k_{x}^{2} k_{y}^{2} .
\end{aligned}
$$

At zero momentum we find for the gap [1]

$$
\hbar \omega_{\mathbf{0}}=\frac{q^{2} s E_{\mathrm{R}} Q(q \xi)}{8 n a_{s}},
$$

corresponding to an eigenvector $\propto(1, i)$. From this eigenvector we therefore conclude that the zero-momentum mode physically corresponds to a precession of the vortices around the maxima of the optical lattice potential, as expected.

\section{The gap}

If one tunes the strength of the periodic potential, the vortex lattice changes, depending on the filling $\nu$. The energy gap of the collective modes is different in the three vortex lattice phases. To summarize the results on the gap, we have

$$
\hbar \omega_{\mathbf{0}}= \begin{cases}0.5 \eta \frac{d_{z}}{8 a_{s}}[q \xi Q(q \xi)]^{2} s E_{\mathrm{R}} & \text { hexagonal vortex lattice } \\ 0 & \text { half-pinned vortex lattice } \\ (q \xi)^{2} Q(q \xi) s E_{\mathrm{R}} / \pi & \text { fully-pinned vortex lattice }\end{cases}
$$




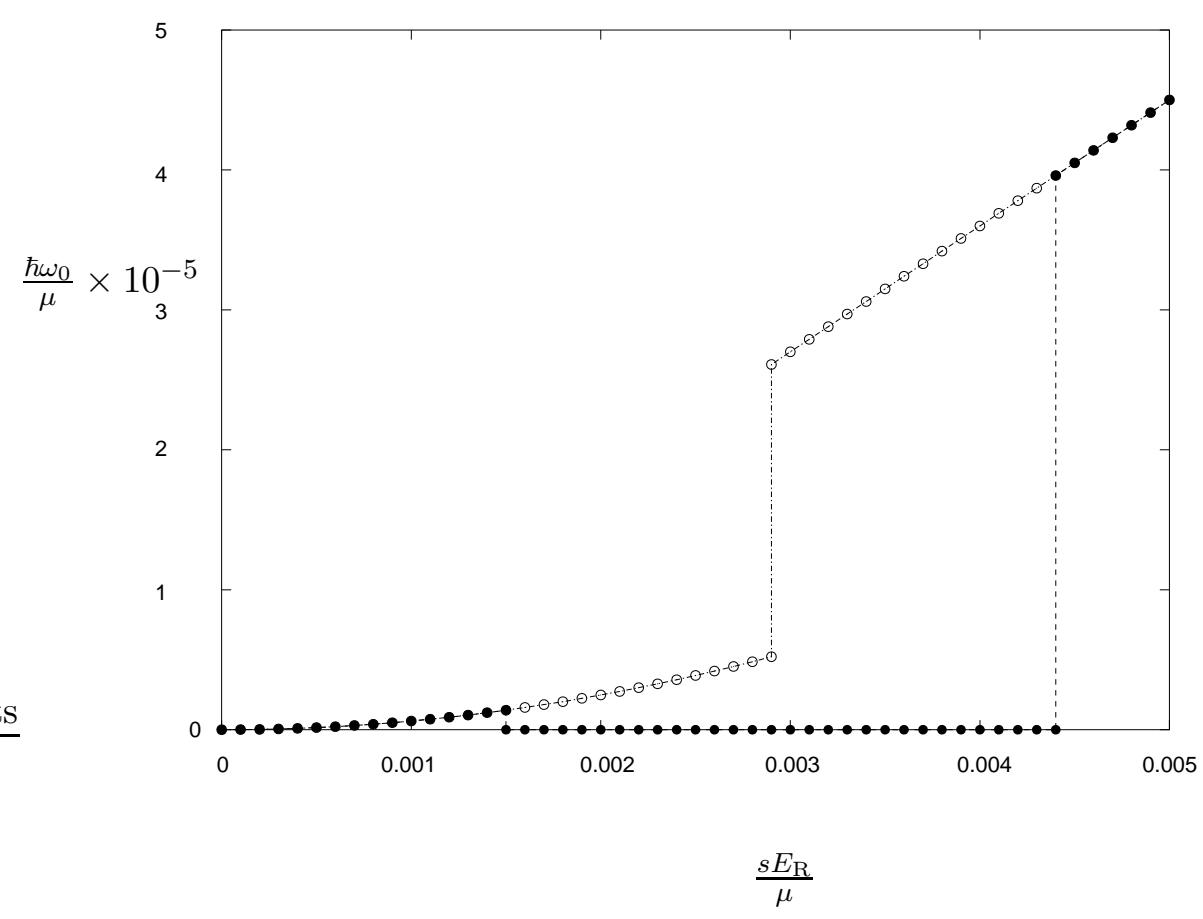

FIG. 4: The energy gap to the collective modes of a vortex lattice in the presence of a periodic optical potential as function of the lattice strength, for $\nu=\frac{1}{4}$ (open circles) and $\nu=\frac{1}{5}$ (filled circles). We used $\eta=0.64\left(\frac{s E_{\mathrm{R}}}{\mu}\right)$ (see [72]) and $q \xi=.05$.

It is clear that the gap in the hexagonal phase is much smaller than the gap in the fullypinned vortex phase. This is because in the hexagonal phase, the non-zero contribution comes entirely from the displacements of the vortices from the equilibrium positions of the hexagonal lattice. The gap is then of second order in $s E_{\mathrm{R}}$, since $\eta \propto s E_{\mathrm{R}}$.

For the half-pinned and fully-pinned vortex lattices, there is no second order contribution to the gap. This is because in these phases the vortices are located on minima and saddle points of the pinning potential. The fact that the energy gap in the half-pinned phases is zero, relies on the fact that we consider infinite vortex lattices. In a trapped system there will be a gap to collective excitations, that becomes smaller with increasing system size.

In Fig. (4) we display the gap as function of the dimensionless parameter $\frac{s E_{\mathrm{R}}}{\mu}$ for the cases $\nu=\frac{1}{4}$ and $\nu=\frac{1}{5}$ which we have considered in this paper. It is clearly seen that the gap has a discontinuity if $\frac{s E_{\mathrm{R}}}{\mu}$ is tuned through a phase boundary. It must be emphasized that in the Abrikosov phase only the qualitative features of the behavior of the gap can be deduced from Fig. (44). This is because the gap then depends on the shear modulus $\mu_{H}$ which is a phenomenological constant in our calculations. 
Finally, we would like to comment on the experimental implications of the collective mode spectra we have calculated. Although our calculations contain phenomenological parameters, there are nonetheless some qualitative predictions that could be tested experimentally. First of all, for the half-pinned and fully-pinned vortex lattices the collective mode spectrum is anisotropic, i.e., the frequency of the collective modes depends on the direction of propagation. By performing the same experiment as Coddington et al. [19], in which the collective modes are excited by a perturbation at the center of the condensate, one could probe this anisotropy. Another prediction of our theory is that the collective modes are in general gapped in the presence of the vortex lattice. An exception is the triangular halfpinned vortex lattice, which has a gapless mode, corresponding to the translation of the vortices in one direction. (For the illustration in Fig. 2 this direction is the $x$-direction.) For the propagation of the modes in the other direction there will be a gap. This strong anisotropy should be experimentally observable by the above-mentioned experiment. The excitations of the fully-pinned vortex lattice are also gapped. As mentioned before, the zeromomentum mode corresponds in this case to a simultaneous in-phase precession of all the vortices around the maxima of the optical potential. This mode could be excited by slightly displacing the optical lattice. Because this zero-momentum mode of the vortex lattice does not have shear or compression, it does not depend on the elasticity constants, which are phenomenological in our calculation. Hence Eq. (60) should give an accurate prediction for the gap in this case, which is directly experimentally verifiable.

\section{CONCLUSIONS}

In this paper, we have presented a method to determine the ground state phase diagram of vortices in a Bose-Einstein condensate in an optical lattice, thereby extending previous work [37] to an arbitrary number of vortices per unit cell of the optical lattice, the so-called filling factor. The vortices arrange themselves in various patterns, depending filling factor and the optical lattice strength. Generally, we find three vortex phases, $i$ ) a fully-pinned phase in which each vortex is pinned to a maximum of the periodic potential, ii) a phase in which half of the vortices are pinned to maxima of the optical lattice and iii) a phase in which none of the vortices are pinned, and the structure of the vortex lattice is determined

by the interactions. The structure of the unpinned phase is always hexagonal, whereas the 
structure of the half-pinned phase is triangular. We have discussed several distinct filling factors explicitly, to demonstrate the above generic features. We calculated the dispersion of low-lying phonon modes of the vortex lattice for each of these phases. In the case of the half-pinned and fully-pinned vortex lattice we find that the collective mode spectrum is anisotropic. Furthermore, in the unpinned and fully-pinned phase the collective modes are gapped. Both features should be observable experimentally, and we have outlined possible experiments to probe the collective modes.

There are several interesting directions for further investigation of the influence of a periodic potential on the physics of rotating Bose-Einstein condensates. For instance, it would be interesting to consider more strongly-correlated regimes that occur at fast rotation 62], and to study the effects of the periodic optical potential on the melting of the vortex lattice [60]. One would expect that in this regime the effect of quantum fluctuations, i.e., quantum tunnelling of the vortices through the potential barriers of the pinning potential, becomes important. Aspects of this were studied by Sørensen et al. [63], who showed that for ultra low particle and vortex density the ground state of rotating bosons in a periodic potential is a Laughlin liquid. It would be challenging to investigate the system with high particle and vortex density and a large number of vortices per boson.

Yet another interesting possibility for future work is to study a rotating spinor condensate in the presence of a periodic potential. Rotating spinor condensates are expected to form spin-textures (skyrmions) [64, 65] and regular lattices thereof [66, 67], analogous to the formation of vortices in a single component condensate. The pinning effects in each spin component of the condensate caused by the periodic potential will further enrich the phase diagram in these systems.

We would like to thank M. Hafezi, R. Hagemans, F.J.M. van Lankvelt, A.H. MacDonald, P. Pedri, V. Schweikhard, K. Schoutens, and H.T.C. Stoof for useful discussions. This research was supported by the National Science Foundation under grant DMR-0115947 (R.A.D.) and by the Netherlands Organization for Scientific Research, NWO (J.W.R.).

[1] L. Onsager, Nuovo Cimento Suppl. 6, 249 (1949)

[2] R.P. Feynman in Progress in Low Temperature Physics, edited by C.J. Gorter (North Holland, 
Amsterdam, 1955), Vol. 1, p. 17.

[3] A.A. Abrikosov, Zh. Eksp. Teor. Fiz. 32, 1142 (1957) [Sov. Phys. - JETP 5, 1174 (1957)].

[4] D. Cribier, B. Jacrot, L.M. Rao, and B. Farnoux, Phys. Lett. 9, 106 (1964).

[5] U. Essmann and H. Traeuble, Phys. Lett. A 24, 526 (1967).

[6] V.K. Tkachenko, Zh. Eksp. Teor. Fis. 49, 1875 (1965) [Sov. Phys. — JETP 22, 1282 (1965)].

[7] S.J. Tsakadze, Fiz. Nizk. Temp. 4, 148 (1978) [Sov. J. Low. Temp. Phys. 4, 72 (1978)].

[8] E.J. Yarmchuk, M.J.V. Gordon, and R.E. Packard, Phys. Rev. Lett. 43, 214 (1979).

[9] V.K. Tkachenko, Zh. Eksp. Teor. Fis. 50, 1573 (1966) [Sov. Phys. — JETP 23, 1049 (1966)].

[10] M.H. Anderson, J.R. Ensher, M.R. Matthews, C.E. Wieman, and E.A. Cornell, Science 269, 198 (1995).

[11] M. R. Matthews, B. P. Anderson, P. C. Haljan, D. S. Hall, C. E. Wieman, and E. A. Cornell, Phys. Rev. Lett. 83, 2498 (1999).

[12] B.P. Anderson, P. C. Haljan, C. E. Wieman, and E. A. Cornell, Phys. Rev. Lett. 85, 2857 (2000).

[13] P. Rosenbusch, V. Bretin, and J. Dalibard, Phys. Rev. Lett. 89, 200403 (2002).

[14] I. Coddington, P. C. Haljan, P. Engels, V. Schweikhard,S. Tung, and E. A. Cornell, Phys. Rev. A 70, 063607 (2004).

[15] K. W. Madison, F. Chevy, W. Wohlleben, and J. Dalibard, Phys. Rev. Lett. 84, 806 (2000).

[16] J. R. Abo-Shaeer, C. Raman, J. M. Vogels, and W. Ketterle, Science 292, 476 (2001).

[17] P. C. Haljan, I. Coddington, P. Engels, and E. A. Cornell, Phys. Rev. Lett. 87, 210403 (2001).

[18] E. Hodby, G. Hechenblaikner, S. A. Hopkins, O. M. Maragò, and C. J. Foot, Phys. Rev. Lett. 88, 010405 (2002).

[19] I. Coddington, P. Engels, V. Schweikhard, and E. A. Cornell, Phys. Rev. Lett. 91, 100402 (2003).

[20] G. Baym, Phys. Rev. Lett. 91, 110402 (2003).

[21] W. I. Glaberson and R. J. Donnelly, Phys. Rev. 141, 208 (1966).

[22] James C. Weaver, Phys. Rev. A 6, 378 (1972).

[23] A.T. Fiory, A.F. Hebard, and S. Somekh, Appl. Phys. Lett. 32, 73 (1978).

[24] M. Baert, V. V. Metlushko, R. Jonckheere, V. V. Moshchalkov, and Y. Bruynseraede, Phys. Rev. Lett. 74, 3269 (1995).

[25] A. Castellanos, R. Wordenweber, G. Ockenfuss, A. v.d. Hart, and K. Keck, Appl. Phys. Lett. 
71, 962 (1997).

[26] J. I. Marín, M. Vélez, J. Nogués, and Ivan K. Schuller, Phys. Rev. Lett. 79, 1929 (1997).

[27] David J. Morgan and J. B. Ketterson, Phys. Rev. Lett. 80, 3614 (1998).

[28] D. R. Nelson and B. I. Halperin, Phys. Rev. B 19, 2457 (1979).

[29] E. Brézin, D. R. Nelson, and A. Thiaville, Phys. Rev. B 31, 7124 (1985).

[30] G. Blatter, M. V. Feigel'man, V. B. Geshkenbein, A. I. Larkin, and V. M. Vinokur, Rev. Mod. Phys. 66, 1125 (1994).

[31] L. Radzihovsky, Phys. Rev. Lett. 74, 4923 (1995).

[32] C. Reichhardt, C. J. Olson, and Franco Nori, Phys. Rev. Lett. 78, 2648 (1997).

[33] A. B. Kolton, Daniel Domínguez, and N. Gronbech-Jensen, Phys. Rev. Lett. 83, 3061 (1999).

[34] C. Dasgupta and O. T. Valls, Phys. Rev. B 66, 064518 (2002).

[35] V. Zhuravlev and T. Maniv, Phys. Rev. B 68, 174507 (2003).

[36] W. V. Pogosov, A. L. Rakhmanov, and V. V. Moshchalkov, Phys. Rev. B 67, 014532 (2003).

[37] J.W. Reijnders and R.A. Duine, Phys. Rev. Lett. 93, 060401 (2004).

[38] M. Greiner, O. Mandel, T. Esslinger, T. W. Hänsch, and I. Bloch, Nature 415, 39 (2002).

[39] M.P.A. Fisher, P. B. Weichman, G. Grinstein, and D. S. Fisher, Phys. Rev. B 40, 546 (1989).

[40] D. Jaksch, C. Bruder, J.I. Cirac, C. W. Gardiner and P. Zoller, Phys. Rev. Lett. 81, 3108 (1998).

[41] P.G. Kevrekidis, R. Carretero-González, G. Theocharis, D.J. Frantzeskakis and B.A. Malomed, J. Phys. B: At. Mol. Opt. Phys. 36, 3467 (2003).

[42] A.B. Bhattacherjee, O. Morsch and E. Arimondo, J. Phys. B: At. Mol. Opt. Phys. 37, 2355 (2004).

[43] D. Boiron, A. Michaud, J. M. Fournier, L. Simard, M. Sprenger, G. Grynberg, and C. Salomon, Phys. Rev. A 57, R4106 (1998).

[44] R. Dumke, M. Volk, T. Mther, F. B. J. Buchkremer, G. Birkl, and W. Ertmer, Phys. Rev. Lett. 89, 097903 (2002).

[45] Z. Chen and K. McCarthy, Opt. Lett. 27, 2019 (2002).

[46] R. Newell, J. Sebby, and T.G. Walker, Opt. Lett. 28, 1266 (2003).

[47] H. Pu, L.O. Baksmaty, S. Yi and N.P. Bigelow, Phys. Rev. Lett. 94, 190401 (2005).

[48] See A.L. Fetter and A.A. Svidzinsky, J. Phys.: Condens. Matter 13, R135 (2001), and references therein. 
[49] See, for example, H. Kleinert, Gauge fields in condensed matter (World Scientific, Singapore, 1989).

[50] L.J. Campbell, M.M. Doria and J.B. Kadtke, Phys. Rev. A 39, 5436 (1989).

[51] E. J. Mueller and T.-L. Ho, Phys. Rev. Lett. 88, 180403 (2002).

[52] K. Kasamatsu, M. Tsubota and M. Ueda, Phys. Rev. Lett. 91, 150406 (2003).

[53] V. Schweikhard, I. Coddington, P. Engels, S. Tung and E.A. Cornell, Phys. Rev. Lett. 93, 210403 (2004).

[54] T.-L. Ho and V. B. Shenoy, Phys. Rev. Lett. 77, 3276 (1996).

[55] B.D. Esry, C.H. Greene, J.P. Burke,Jr., and John L. Bohn, Phys. Rev. Lett. 78, 3594 (1997).

[56] E. Timmermans, Phys. Rev. Lett. 81, 5718 (1998).

[57] L.D. Landau and E.M. Lifschitz, Theory of Elasticity (Pergamon Press, New York, 1970).

[58] Lynn Bonsall and A.A. Maradudin, Phys. Rev. B 15, 1959 (1977).

[59] M. Abolfath, K. Mullen, and H. T. C. Stoof, Phys. Rev. B 63, 075315 (2001).

[60] J. Sinova, C. B. Hanna, and A. H. MacDonald, Phys. Rev. Lett. 89, 030403 (2002).

[61] See E. B. Sonin, Rev. Mod. Phys. 59, 87 (1987), and references therein.

[62] Marco Polini, Rosario Fazio, A. H. MacDonald, and M. P. Tosi, cond-mat/0501375

[63] A.S. Sørensen, E. Demler and M.D. Lukin, Phys. Rev. Lett. 94, 086803 (2005).

[64] U. Al Khawaja and H.T.C. Stoof, Nature 411, 918 (2001).

[65] J. Ruostekoski and J. R. Anglin, Phys. Rev. Lett. 86, 3934 (2001).

[66] T. Kita, T. Mizushima and K. Machida, Phys. Rev. A 66, 061601 (2002).

[67] J.W. Reijnders, F.J.M. van Lankvelt, K. Schoutens and N. Read, Phys. Rev. A 69, 023612 (2004).

[68] See also Ref. 41], which treats the limit of a small number of condensate atoms, as opposed to the Thomas-Fermi regime we consider here.

[69] Apart from breaking the translational symmetry, such a triangular vortex lattice also breaks a discrete symmetry, because there are several distinct possibilities to form the triangular lattice configuration. In this particular case the discrete symmetry is $Z_{4}$. This additional symmetry breaking implies the existence of domain walls between triangular vortex lattices that are oriented differently. We do not consider such configurations here.

[70] Note that if we rotate the vortex lattice over an angle $\pi / 4$, we we would find the the dynamical matrix is proportional to $\delta_{\alpha, x}$ instead of $\delta_{\alpha, y}$. (See also [69].) 
[71] Note that in Eq. (10) of Ref. 37] there should be an additional factor of $2 \pi$.

[72] We obtained this estimate using the expression for the shear modulus following from hydrodynamic theory. We have $e_{\text {shear }}=\mu_{H} A=\mu_{H}^{3 \mathrm{D}} d_{z} A=\frac{n \hbar \Omega}{8} d_{z} \pi \ell^{2}$, with $\ell^{2}=\frac{\hbar}{M \Omega}$. The ratio between the chemical potential $\mu=g n$ and $e_{\text {shear }}$ is constant, $\frac{e_{\text {shear }}}{\mu}=\frac{d_{z}}{32 a_{s}}$. We then use $a_{s} \approx 5 \mathrm{~nm}$ for ${ }^{87} \mathrm{Rb}$ and $d_{z} \approx 10 \mu \mathrm{m}$ to find $\eta=\frac{s E_{\mathrm{R}}}{e_{\text {shear }}}=0.64\left[\frac{s E_{\mathrm{R}}}{\mu}\right]$. 